\section{Advancing an HIV vaccine; advancing vaccinology}

\section{Dennis R. Burton}

Of any pathogen, HIV provides perhaps the greatest challenge to successful vaccine development. Nevertheless, progress continued to be made in 2018; new vaccine concepts entered the clinic and new insights were obtained in basic research that will ultimately help to guide rational vaccine design against many 'difficult' pathogens.
There is widespread agreement that eliminating HIV from the human population will require a successful vaccine. However, candidate vaccines evaluated to date have either failed or have shown very modest and debated efficacy. New vaccine trials have been initiated that are based on inducing both antibodies and $\mathrm{T}$ cell immunity, and the results from these trials will become known in the next few years. At the same time, abundant data have shown that broadly neutralizing antibodies (bnAbs) are induced in natural HIV infection and that such antibodies, provided by passive transfer, can both protect from HIV in robust animal models and affect ongoing HIV infection in humans ${ }^{1}$. An important passive bnAb study, the Antibody-Mediated Prevention (AMP) trial ${ }^{2}$, is now fully enrolled and will hopefully provide answers in 2019-2020 as to the levels of bnAbs that protect humans from acquisition of HIV. There are also large ongoing efforts to explore the possibility of using combinations of bnAbs as prophylactics by passive transfer.

Meanwhile, efforts towards the rational design of a bnAb-inducing HIV vaccine have centred on sequential multi-immunogen strategies $^{3}$ (FIG. 1). In other words, unlike, for example, a human papillomavirus vaccine that uses a sequence of immunizations with three identical immunogens, an HIV vaccine may require a sequence of immunizations with different immunogens to guide antibody responses from naive B cells to mature bnAbs. Key elements of the most promising strategies include the notion of designing immunogens that are templated from known bnAbs (reverse vaccinology 2.0); activating particular bnAb-producing cell precursors and then shepherding their affinity maturation to generate mature bnAbs (germline targeting or lineage-based design); the use of recombinant stabilized HIV Envelope (Env) trimers (particularly those known as SOSIP trimers) as the basis for many immunogens; and the deployment of humanized animal models to evaluate immunogens.

I focus here on a small number of papers from 2018 that, in my opinion, have most advanced insight into how bnAb-based rational $\mathrm{HIV}$ vaccine design can proceed and have contributed most to strategies to design vaccines to other difficult pathogens such as malaria and dengue virus, which present problems for classical approaches.

Rational HIV vaccine design targets bnAb-binding sites identified on the HIV Env spike protein (which comprises three gp120s and three gp41s in a trimer of non-covalently linked heterodimers), such as the CD4-binding site (CD4bs) and the gp120-gp41 interface region. Kwong, Mascola and colleagues previously identified a bnAb specific for the interface region, known as VRC34, that recognizes an epitope including the eight amino-terminal residues of fusion peptide (FP; residues 512-527 of gp41 that mediates membrane fusion). They designed immunogens based on FP linked to a carrier protein and immunized mice to generHIV strains ${ }^{4}$. Following sequence analysis and structural studies, they generated improved immunogens and immunization strategies, now involving both FP and a SOSIP trimer, to elicit second generation antibodies that neutralize up to $31 \%$ of HIV strains in mice. The improved regimes were then tested in non-human primates (NHPs). At a serum dilution of 1:20, 3 of 5 sera from vaccinated NHPs showed neutralization breadth, with 1 serum neutralizing about $22 \%$ of HIV strains well. This is far from ideal, but it is a very promising start given the relative simplicity of the immunogens and immunization regime and has fuelled considerable new interest in $\mathrm{FP}$ as an HIV vaccine target.

an HIV vaccine may require a sequence of immunizations with different immunogens to guide antibody responses from naive $B$ cells to mature bnAbs

The CD4bs is perhaps the most favoured bnAb target given the combination of potency and breadth shown by CD4bs-specific bnAbs. The VRC01 class of CD4bs-specific bnAbs uses a single immunoglobulin heavy $(\mathrm{H})$ chain variable $(\mathrm{V})$ germline gene segment (VH1-2) and is therefore particularly amenable for the design of immunogens to induce this class of bnAb. A VRC01-class-germlinetargeting immunogen, known as eOD-GT8 (engineered outer domain of gp120germline targeting generation 8 ; which is a self-assembling nanoparticle composed of an engineered outer domain from HIV gp120 fused to a lumazine synthase protein scaffold), has been designed by Schief and colleagues using computational modelling and yeast display selection ${ }^{5}$. eOD-GT8 entered the clinic in 2018 based on a series of successes in animal model studies. An elegant model study by Crotty and colleagues ${ }^{6}$ used a series of eOD constructs to explore the conditions ate antibodies that neutralized up to $10 \%$ of

\section{Key advances}

- The fusion peptide of HIV Envelope (Env) has emerged as a potential vaccine target.

- Naive B cell precursor frequencies and antigen affinities are crucial in considering the likelihood of success with a germline-targeting immunogen.

- Off-target responses to immunogens can be reduced by glycan masking of irrelevant epitopes.

- Neutralization, but not other immune parameters, correlates with HIV trimer vaccine-induced protection against viral challenge in a monkey model.

- Electron microscopy is a rapid method to determine the specificities present in a polyclonal antibody response following vaccination or infection. 
for successful germline targeting. Briefly, naive B cells with VRC01 class B cell receptors (BCRs) were adoptively transferred into wild-type mice at different frequencies and then the mice were immunized with eOD constructs of differing affinities for the naive VRC01 BCR. The key finding was that B cell precursor frequency and antigen affinity each determine competitive fitness of $B$ cells in germinal centres. Thus, at a naive $\mathrm{B}$ cell precursor frequency of 1 in $10^{3}$, eOD constructs of low affinity successfully activated naive B cells and generated B cell memory. At a frequency of 1 in $10^{6}$, only a germline-targeting construct of high physiological affinity was effective. A similar finding was reported in a parallel study $^{7}$. Naive B cell precursor frequencies and antigen affinities are thus expected to be crucial considerations for germline targeting in humans. As eOD-GT8 entered the clinic, Mascola and colleagues ${ }^{8}$ showed how the germline-targeting properties of the immunogen could be improved by reducing off-target antibody responses. They used extra glycans to mask irrelevant epitopes and change immunodominance to focus responses to the desired CD4bs epitope. This approach is likely to find general utility.

Sequential immunization strategies (FIG. 1) are often envisaged to start with germline targeting and finish with recombinant Env trimers that mimic the native virion trimer (for example, SOSIP trimers). The prototype

\section{ce \\ Naive B cell precursor frequencies and antigen affinities are thus expected to be crucial considerations for germline targeting in humans}

Env is the BG505 SOSIP.664 trimer (soluble, cleaved SOSIP.664 gp140 trimers based on the HIV strain BG505), which entered clinical trials in 2018. In NHPs, immunization with BG505 SOSIP.664 generated high titres of autologous serum neutralizing antibody in some animals and low titres in other animals? Subsequent challenge with the SHIV BG505 (a simian/human immunodeficiency virus (SHIV) construct expressing surface HIV BG505 trimers and exhibiting neutralization resistance characteristic of circulating HIV (a so-called tier 2 virus)) showed a tight correlation between serum neutralization titres at the time of challenge and protection from repeated medium dose intrarectal challenge. Animals with high-titre autologous serum neutralizing antibody were substantially protected from infection. Antibody-dependent cellular cytotoxicity and $\mathrm{T}$ cell responses did not correlate with protection. It will be of great interest to compare the observed protective titres induced by SHIV in NHPs with those from the AMP study as a reflection of the utility of the SHIV-NHP model. Similarly,
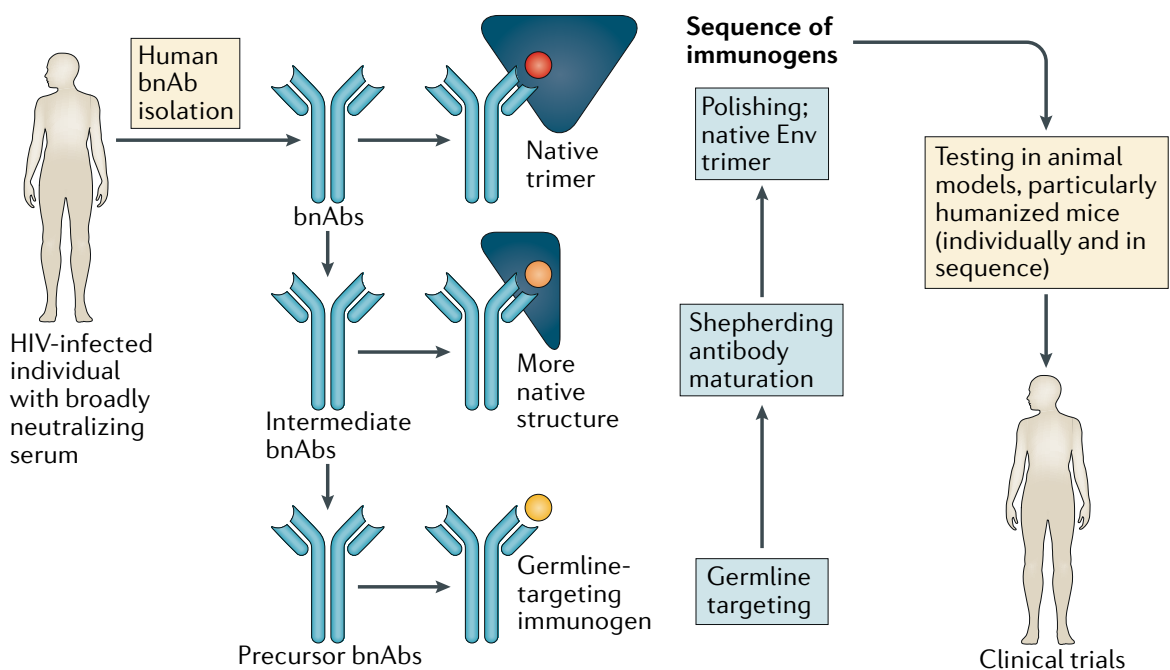

Fig. 1 | A sequential HIV immunization strategy. Broadly neutralizing antibodies (bnAbs) isolated from infected individuals provide proof of principle that a bnAb-based HIV vaccine is possible. The bnAbs define vaccine targets on native HIV Envelope (Env) trimers (red). Inferred precursors of the bnAbs (often referred to as inferred germline bnAbs) do not generally bind to native trimers, requiring the design of immunogens to activate precursor bnAb B cells (germline-targeting immunogens, for example, eOD-GT8 (yellow)). In order to shepherd the antibody response towards mature bnAbs, a series of immunogens with increasing native trimer-like features are used; these immunogens can be validated by antibodies derived from bnAbs but of lower potency or breadth. Finally, immunization is carried out with native-like trimers such as SOSIP trimers. Immunogen testing uses animal models expressing bnAb precursor $\mathrm{B}$ cells (knock-in mice) or human antibody repertoires before moving into the clinic. since the ongoing SOSIP clinical trial uses the same BG505 SOSIP.664 immunogen as used in the NHP study, it will be important to compare the magnitude of neutralization and the epitopes targeted in humans and NHPs to further gauge the utility of the NHP model.

The polyclonal antibody response to SOSIP trimer immunization of rabbits was recently analysed by Hangartner, Ward and colleagues ${ }^{10}$ by a rapid new electron microscopy approach. Responses to known and novel epitopes were described, in some cases at sub-nanometre resolution, and the approach was used to provide a semi-quantitative map of the complex antibody response to HIV Env. One can envisage that the approach will find widespread application in studying antibody responses to vaccination and infection, especially for comparison of antibody responses in animal models to those in humans.

Dennis R. Burton (iD ${ }^{1,2}$

'Department of Immunology and Microbiology, the Scripps Center for HIVIAIDS Vaccine Immunology and Immunogen Discovery (CHAVI-ID), International AIDS Vaccine Initiative Neutralizing Antibody Center, Scripps Research, La Jolla, CA, USA

${ }^{2}$ Ragon Institute of Massachusetts General Hospital, Massachusetts Institute of Technology and Harvard, Cambridge, MA, USA.

e-mail:burton@scripps.edu https:/doi.org/10.1038/s41577-018-0103-6

Escolano, A., Dosenovic, P. \& Nussenzweig, M. C. Progress toward active or passive HIV-1 vaccination. J. Exp. Med. 214, 3-16 (2017).

2. HIV Prevention Trials Network \& HIV Vaccine Trials Network. AMP HIV Prevention Study. AMP Study https://ampstudy.org (2018).

3. Andrabi, R., Bhiman, J. N. \& Burton, D. R. Strategies for a multi-stage neutralizing antibody-based HIV vaccine. Curr. Opin. Immunol. 53, 143-151 (2018).

4. Xu, K. et al. Epitope-based vaccine design yields fusion peptide-directed antibodies that neutralize diverse strains of HIV-1. Nat. Med. 24, 857-867 (2018).

5. Jardine, J. G. et al. HIV-1 broadly neutralizing antibody precursor B cells revealed by germline-targeting immunogen. Science 351, 1458-1463 (2016).

6. Abbott, R. K. et al. Precursor frequency and affinity determine $B$ cell competitive fitness in germinal centers, tested with germline-targeting HIV vaccine immunogens. Immunity 48, 133-146 (2018).

7. Dosenovic, P. et al. Anti-HIV-1 B cell responses are dependent on $B$ cell precursor frequency and antigen-binding affinity. Proc. Natl Acad. Sci. USA 115, 4743-4748 (2018).

8. Duan, $H$. et al. Glycan masking focuses immune responses to the HIV-1 CD4-binding site and enhances elicitation of VRC01-class precursor antibodies. Immunity 49, 301-311 (2018).

9. Pauthner, M. P. et al.Vaccine-induced protection from homologous tier 2 SHIV challenge in nonhuman primates depends on serum-neutralizing antibody titers. Immunity https://doi.org/10.1016/j.immuni. 2018.11.011 (2018)

10. Bianchi, M. et al. Electron-microscopy-based epitope mapping defines specificities of polyclonal antibodies elicited during HIV-1 BG505 envelope trimer immunization. Immunity 49, 288-300 (2018).

Acknowledgements

Thanks to S. Crotty, L. Hangartner, J. Mascola and A. Ward for comments on the manuscript and to the National Institute of Allergy and Infectious Diseases (NIAID), International AIDS Vaccine Initiative (IAVI) and the Bill \& Melinda Gates Foundation for financial support.

\section{Competing interests}

The author declares no competing interests. 